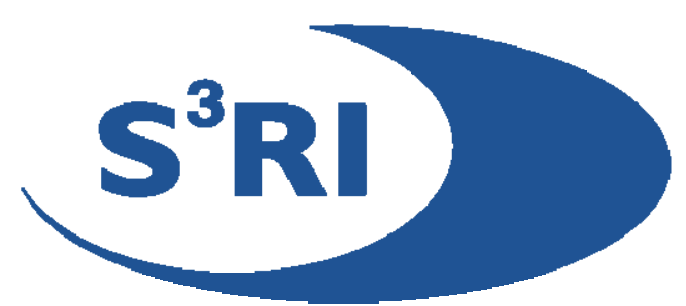

\title{
MINIMUM AREA CONFIDENCE SET OPTIMALITY FOR CONFIDENCE BANDS IN SIMPLE LINEAR REGRESSION
}

\author{
W. LIU, A. J. HAYTER
}

\begin{abstract}
The average width of a simultaneous confidence band has been used by several authors (e.g. Naiman, 1983, 1984, Piegorsch, 1985a) as a criterion for the comparison of different confidence bands. In this paper, the area of the confidence set corresponding to a confidence band is used as a new criterion. For simple linear regression, comparisons have been carried out under this new criterion between hyperbolic bands, two-segment bands, and three-segment bands, which include constant width bands as special cases. It is found that if one requires a confidence band over the whole range of the covariate, then the best confidence band is given by the Working \& Hotelling hyperbolic band. Furthermore, if one needs a confidence band over a finite interval of the covariate, then a restricted hyperbolic band can again be recommended, although a three-segment band may be very slightly superior in certain cases.
\end{abstract}

\section{Southampton Statistical Sciences Research Institute Methodology Working Paper M07/02}




\title{
Minimum area confidence set optimality for
}

\section{confidence bands in simple linear regression}

\author{
W. Liu \\ $S^{3} R I$ and School of Mathematics, University of Southampton, Southampton, UK \\ W.Liu@maths.soton.ac.uk
}

A.J. Hayter

Department of Statistics and Operations Technology, University of Denver, USA

Anthony.Hayter@du.edu

Revised August 2006

\begin{abstract}
Summary
The average width of a simultaneous confidence band has been used by several authors (e.g. Naiman, 1983, 1984, Piegorsch, 1985a) as a criterion for the comparison of different confidence bands. In this paper, the area of the confidence set corresponding to a confidence band is used as a new criterion. For simple linear regression, comparisons have been carried out under this new criterion between hyperbolic bands, two-segment bands, and three-segment bands, which include constant width bands as special cases. It is found that if one requires a confidence band over the whole range of the covariate, then
\end{abstract}


the best confidence band is given by the Working \& Hotelling hyperbolic band.

Furthermore, if one needs a confidence band over a finite interval of the covariate, then a restricted hyperbolic band can again be recommended, although a three-segment band may be very slightly superior in certain cases.

Key words: Simple Linear Regression; Confidence Bands; Confidence Sets; Probability Inequalities; Minimum Area.

\section{Introduction}

Suppose that data $\left(x_{i}, y_{i}\right)$ are available which are modelled as

$$
y_{i}=b_{0}+b_{1}\left(x_{i}-\bar{x}\right)+\epsilon_{i}, \quad i=1, \cdots, n
$$

where $\bar{x}=\sum_{i=1}^{n} x_{i} / n$, the errors $\epsilon_{i}$ are independently distributed as $N\left(0, \sigma^{2}\right)$ random variables, and $b_{0}, b_{1}$ and $\sigma^{2}$ are unknown parameters. Let $X$ denote the usual design matrix, whose $i$ th row is given by $\left(1, x_{i}-\bar{x}\right), i=1, \cdots, n$. Then $\left(X^{T} X\right)^{-1}$ is a $2 \times 2$ diagonal matrix with the first and second diagonal elements given by $1 / n$ and $1 / s_{x x}=$ $1 / \sum_{i=1}^{n}\left(x_{i}-\bar{x}\right)^{2}$ respectively. Let $U$ be the square root matrix of $\left(X^{T} X\right)^{-1}$. Denote the least square estimators of $\boldsymbol{b}=\left(b_{0}, b_{1}\right)^{T}$ and $\sigma$ by $\hat{\boldsymbol{b}}=\left(\hat{b}_{0}, \hat{b}_{1}\right)^{T}$ and $\hat{\sigma}$ respectively. Then $\hat{\boldsymbol{b}} \sim N_{2}\left(\boldsymbol{b}, \sigma^{2}\left(X^{T} X\right)^{-1}\right), \hat{\sigma} / \sigma \sim \sqrt{\chi_{k}^{2} / k}$ where $k=n-2$, and $\hat{\boldsymbol{b}}$ and $\hat{\sigma}$ are independent random variables.

\subsection{Confidence Bands}

In this article we consider only those confidence bands $(l(x-\bar{x}), u(x-\bar{x}))$ for $b_{0}+b_{1}(x-\bar{x})$ over an interval $x-\bar{x} \in(a, A)$ that have confidence level equal to $1-\alpha$ :

$$
\inf _{-\infty<b_{0}, b_{1}<\infty, \sigma>0} \mathrm{P}\left\{l(x-\bar{x}) \leq b_{0}+b_{1}(x-\bar{x}) \leq u(x-\bar{x}), \forall x-\bar{x} \in(a, A)\right\}=1-\alpha .
$$


A confidence band provides useful information on where the true but unknown regression line lies; a straight line is a plausible candidate for the unknown regression line if and only if it is contained completely inside the confidence band. There are several recent papers that consider applications of confidence bands. For example, Al-Saidy et al. (2003) and Piegorsch et al. (2005) use confidence bands in risk analysis, while Spurrier (1999) and Liu et al. (2004) use confidence bands for simultaneous comparisons of several linear regression models.

The most commonly used confidence bands are hyperbolic bands (Working \& Hotelling, 1929, Scheffé, 1953, Wynn and Bloomfield, 1971, Uusipaikka, 1983), two-segment bands (Graybill and Bowden, 1967), and three-segment bands (Bowden and Graybill, 1966), even though there are still other forms of confidence bands (e.g. Bowden, 1970, Naiman, 1987, Piegorsch, et al., 2000). All of these two-sided bands are symmetric about the estimated regression line $\hat{b}_{0}+\hat{b}_{1}(x-\bar{x})$ so that

$$
l(x-\bar{x})=\hat{b}_{0}+\hat{b}_{1}(x-\bar{x})-H(x-\bar{x}), \quad u(x-\bar{x})=\hat{b}_{0}+\hat{b}_{1}(x-\bar{x})+H(x-\bar{x})
$$

where $H(x-\bar{x})$ is the half width of the band at $x-\bar{x}$. If $v(u)=1 / n+u^{2} / s_{x x}$ then a hyperbolic band has

$$
H_{h}(x-\bar{x})=c_{h} \hat{\sigma} \sqrt{v(x-\bar{x})}
$$

where the critical constant $c_{h}$ is chosen so that the confidence level is equal to $1-\alpha$. If $(a, A)=(-\infty, \infty)$ then $c_{h}=\sqrt{2 f_{2, k}^{\alpha}}$ where $f_{2, k}^{\alpha}$ is the upper $\alpha$ point of the $F$ distribution with 2 and $k=n-2$ degrees of freedom. This band was originally proposed by Working \& Hotelling (1929) although it is usually referred to as the Scheffé $(1953,1959)$ band. When $(a, A)$ is a finite interval, the computation of $c_{h}$ is considered in Wynn and Bloomfield (1971) and Uusipaikka (1983).

A two-segment band has

$$
H_{2}(x-\bar{x})=c_{2,1} \hat{\sigma} / \sqrt{n}+c_{2,2}|x-\bar{x}| \hat{\sigma} / \sqrt{s_{x x}}, \quad x-\bar{x} \in(-\infty, \infty)
$$


where the critical constants $c_{2,1}$ and $c_{2,2}$ are chosen so that the confidence level in (1.1) with $a=-\infty$ and $A=\infty$ is equal to $1-\alpha$. The computation of $c_{2,1}$ and $c_{2,2}$ is discussed in Graybill and Bowden (1967). Notice that conceptually, this two-segment band is constructed by putting two-sided bounds on both $b_{0}$ and $b_{1}$.

A three-segment band has

$$
H_{3}(x-\bar{x})=\frac{\hat{\sigma}}{A-a}\left\{(x-\bar{x}-a) c_{3,1} \sqrt{v(A)}+(A-x+\bar{x}) c_{3,2} \sqrt{v(a)}\right\}, \quad x-\bar{x} \in(a, A)
$$

where the critical constants $c_{3,1}$ and $c_{3,2}$ are chosen so that the confidence level is equal to $1-\alpha$. The calculation of $c_{3,1}$ and $c_{3,2}$ is considered in Bowden and Graybill (1966). When $c_{3,2} \sqrt{v(a)}=c_{3,1} \sqrt{v(A)}$, the three-segment band becomes a constant width band over $x-\bar{x} \in(a, A)$ which is also considered by Gafarian (1964). Notice that conceptually, this three-segment band is constructed by putting two-sided bounds on the regression line at both $a$ and $A$. Outside of $(a, A)$ the bands are formed of straight lines corresponding to the diagonal elements of the band region within $(a, A)$, so that the upper and lower bands both consist of three line segments.

\subsection{Average Width Criterion}

Almost all of the previous work on the comparison of confidence bands has used the average-width (AW) as a criterion, which stems from the desire to bound the unknown regression line over $x-\bar{x} \in(a, A)$ as tightly as possible. For example, a condition is given in Naiman (1983) under which the hyperbolic band has a smaller AW than the constantwidth band when $a=-A$. Naiman (1984) also provides a weight function with respect to which the hyperbolic band has the smallest weighted AW when $(a, A)$ is finite. Piegorsch (1985a) finds the weight functions for which the hyperbolic band and the two-segment band have the smallest weighted AW respectively when $(a, A)=(-\infty, \infty)$. The minimax regret criterion of Naiman (1987) and the work of Piegorsch (1985b) are also related to 
the AW of a band.

However, the AW criterion is not without its flaws. For example, if one uses the AW criterion to compare the hyperbolic band with the two-segment band over $(-\infty, \infty)$, then a value larger than one of the quantity

$$
\lim _{U \rightarrow \infty} \frac{\int_{-U}^{U} 2 H_{h}(w) d w /(2 U)}{\int_{-U}^{U} 2 H_{2}(w) d w /(2 U)}=c_{h} / c_{2,2}
$$

favors the two-segment band, while a value smaller than one favors the hyperbolic band. Therefore, any two-segment band with $c_{2,2}<c_{h}$ is better than the hyperbolic band under the AW criterion. Furthermore, among all the two-segment bands that satisfy the confidence level requirement (1.1), the smaller the value of $c_{2,2}$, then the better the confidence band under the AW criterion. The possible range of $c_{2,2}$ under the constraint $(1.1)$ is $(0, \infty)$. When $c_{2,2}$ is very close to $0, c_{2,1}$ is very large, so that the corresponding two-segment band has the gradients of all of the four line segments, $\pm c_{2,2} \hat{\sigma} / \sqrt{s_{x x}}$, very close to zero, and the smallest width at $x-\bar{x}=0$, which is $2 c_{2,1} \hat{\sigma} / \sqrt{n}$, is very large. This two-segment band seems to be of little use for any practical purposes even though it is judged to be quite good under the AW criterion. Also, note that there is no 'optimal' $1-\alpha$ level two-segment band under the AW criterion since $c_{2,2}$ is not allowed to be 0 .

Another drawback of the AW criterion is that it may also give too much weight to the interval on which the confidence band is presented. Note that the three-segment band is completely determined by the pair of $1-\alpha$ simultaneous confidence intervals at $x-\bar{x}=a$ and $x-\bar{x}=A$ :

$$
b_{0}+b_{1} a \in \hat{b}_{0}+\hat{b}_{1} a \pm \hat{\sigma} c_{3,2} \sqrt{v(a)}, \quad b_{0}+b_{1} A \in \hat{b}_{0}+\hat{b}_{1} A \pm \hat{\sigma} c_{3,1} \sqrt{v(A)}
$$


One can extend the three-segment band from the interval $(a, A)$ to the whole line with

$$
H_{3}(x-\bar{x})=\left\{\begin{array}{lll}
\frac{\hat{\sigma}}{A-a}\left\{-(x-\bar{x}-a) c_{3,1} \sqrt{v(A)}+(A-x+\bar{x}) c_{3,2} \sqrt{v(a)}\right\} & \text { if } \quad x-\bar{x} \in(-\infty, a] \\
\frac{\hat{\sigma}}{A-a}\left\{(x-\bar{x}-a) c_{3,1} \sqrt{v(A)}+(A-x+\bar{x}) c_{3,2} \sqrt{v(a)}\right\} & \text { if } \quad x-\bar{x} \in(a, A) \\
\frac{\hat{\sigma}}{A-a}\left\{(x-\bar{x}-a) c_{3,1} \sqrt{v(A)}-(A-x+\bar{x}) c_{3,2} \sqrt{v(a)}\right\} & \text { if } \quad x-\bar{x} \in[A, \infty) .
\end{array}\right.
$$

The restriction of this overall band to any interval $x-\bar{x} \in\left(a^{*}, A^{*}\right)$ that includes the interval $(a, A)$ (i.e. $\left.-\infty \leq a^{*} \leq a<A \leq A^{*} \leq \infty\right)$ still has a confidence level equal to $1-\alpha$. Consequently, the backbone of a three-segment band is the pair of confidence intervals in (1.5), which in turn are determined by the critical constants $\left(c_{3,1}, c_{3,2}\right)$. The range $x-\bar{x} \in\left(a^{*}, A^{*}\right) \supset(a, A)$ plays only a superficial role in presenting the pair of confidence intervals in the form of a confidence band. Hence one would expect that whether a threesegment band is sound should be fundamentally determined by the pair of underlying confidence intervals, and not by the range $\left(a^{*}, A^{*}\right)$. However this is not the case under the AW criterion, because the range $\left(a^{*}, A^{*}\right)$ turns out to be a crucial element. For instance, the AW of the three-segment band over $x-\bar{x} \in\left(a, A^{*}\right)$ is

$$
A W\left(A^{*}, c_{3,1}, c_{3,2}\right):=\frac{1}{A^{*}-a} \int_{a}^{A^{*}} 2 H_{3}(w) d w
$$

Now one can construct another $1-\alpha$ three-segment band from another pair of $1-\alpha$ simultaneous confidence intervals at $x-\bar{x}=a$ and $A$, with critical constants $\left(\tilde{c}_{3,1}, \tilde{c}_{3,2}\right)$ say, such that $A W\left(A_{1}^{*}, c_{3,1}, c_{3,2}\right)<A W\left(A_{1}^{*}, \tilde{c}_{3,1}, \tilde{c}_{3,2}\right)$ but $A W\left(A_{2}^{*}, c_{3,1}, c_{3,2}\right)>A W\left(A_{2}^{*}, \tilde{c}_{3,1}, \tilde{c}_{3,2}\right)$ for some $A<A_{1}^{*}<A_{2}^{*}<\infty$. Therefore, which three-segment band is better under the AW criterion depends critically on the range even though the two pairs of simultaneous confidence intervals underlying the two three-segment bands are fixed.

\subsection{Minimum Area Criterion}

In this article a new criterion for comparing confidence bands is proposed. Note that each $1-\alpha$ level confidence band corresponds to a $1-\alpha$ level confidence set for $\boldsymbol{b}$ in $R^{2}$. See 
Khorasani and Milliken (1979) and Piegorsch (1987) for more details on the correspondence between confidence bands and confidence sets. Let $\boldsymbol{x}=(1, x-\bar{x})^{T}, \boldsymbol{a}=(1, a)^{T}$ and $\boldsymbol{A}=(1, A)^{T}$. Define

$$
R_{h}=\left\{\boldsymbol{b}: \max _{x-\bar{x} \in[a, A]} \frac{\left|(U \boldsymbol{x})^{T} \boldsymbol{b}\right|}{\|U \boldsymbol{x}\|} \leq c_{h}\right\} \subset R^{2} .
$$

Then a hyperbolic band corresponds to the confidence set

$$
C_{h}(\hat{\boldsymbol{b}}, \hat{\sigma})=\left\{\boldsymbol{b}: U^{-1}(\boldsymbol{b}-\hat{\boldsymbol{b}}) / \hat{\sigma} \in R_{h}\right\}
$$

Let

$$
R_{2}=\left\{\boldsymbol{b}:\left|\mathbf{e}_{\mathbf{1}}^{T} \boldsymbol{b}\right| \leq c_{2,1},\left|\mathbf{e}_{\mathbf{2}}^{T} \boldsymbol{b}\right| \leq c_{2,2}\right\} \subset R^{2}
$$

where $\mathbf{e}_{\mathbf{j}}$ is a two-dimensional vector with the $j$ th element equal to one and the other element equal to zero. Then a two-segment band corresponds to the confidence set

$$
C_{2}(\hat{\boldsymbol{b}}, \hat{\sigma})=\left\{\boldsymbol{b}: U^{-1}(\boldsymbol{b}-\hat{\boldsymbol{b}}) / \hat{\sigma} \in R_{2}\right\}
$$

Denote

$$
R_{3}=\left\{\boldsymbol{b}: \frac{\left|(U \boldsymbol{a})^{T} \boldsymbol{b}\right|}{\|U \boldsymbol{a}\|} \leq c_{3,2}, \frac{\left|(U \boldsymbol{A})^{T} \boldsymbol{b}\right|}{\|U \boldsymbol{A}\|} \leq c_{3,1}\right\} \subset R^{2} .
$$

Then a three-segment band corresponds to the confidence set

$$
C_{3}(\hat{\boldsymbol{b}}, \hat{\sigma})=\left\{\boldsymbol{b}: U^{-1}(\boldsymbol{b}-\hat{\boldsymbol{b}}) / \hat{\sigma} \in R_{3}\right\}
$$

The sets $R_{h}, R_{2}$ and $R_{3}$ are illustrated in Figure 1 , in which $R_{h}$ is given by the spindle, $R_{2}$ by the rectangle, and $R_{3}$ by the parallelogram.

The new criterion is based on the area of the confidence set for $\boldsymbol{b}$. The smaller the area of the confidence set, then the better is the corresponding confidence band. This criterion is related to the classical D-optimality in experimental design in that D-optimal designs also minimize the area of the $F$-distribution confidence ellipsoid for $\boldsymbol{b}$ (see e.g. Atkinson and Donev, 1992). Intuitively, each $\boldsymbol{b}$ in a confidence set corresponds to a regression line 
$b_{0}+b_{1}(x-\bar{x})$ which lies completely inside the corresponding confidence bands, and vice versa. Furthermore, each regression line $b_{0}+b_{1}(x-\bar{x})$ that is contained completely inside a confidence band is deemed by this band to be a plausible candidate for the true but unknown regression line. So it is desirable that a confidence band has its corresponding confidence set as small as possible in area. Since the confidence sets $C_{h}, C_{2}$, and $C_{3}$ are generated from sets $R_{h}, R_{2}$, and $R_{3}$ respectively via the same linear transformation $U^{-1}(\boldsymbol{b}-\hat{\boldsymbol{b}}) / \hat{\sigma}$, the comparisons under the new criterion only needs to focus on the areas of $R_{h}, R_{2}$, and $R_{3}$. With the confidence level fixed at $1-\alpha$, a confidence band that has a smaller area $R$ is better, and the one with minimum area (MA) will be considered optimal.

Note that $\boldsymbol{N}=U^{-1}(\boldsymbol{b}-\hat{\boldsymbol{b}}) / \sigma$ has a standard bivariate normal distribution and $\boldsymbol{T}=$ $U^{-1}(\boldsymbol{b}-\hat{\boldsymbol{b}}) / \hat{\sigma}$ has a standard bivariate $t$ distribution (see e.g. Tong, 1990). From the definitions of $R_{h}, R_{2}$, and $R_{3}$,

$$
\mathrm{P}\left\{\boldsymbol{T} \in R_{h}\right\}=\mathrm{P}\left\{\boldsymbol{T} \in R_{2}\right\}=\mathrm{P}\left\{\boldsymbol{T} \in R_{3}\right\}=1-\alpha
$$

so that all three $R$ regions have the same $\boldsymbol{T}$-probability $1-\alpha$.

In Section 2 it is shown that a circular region $R$ has the smallest area among all the regions that have $\boldsymbol{T}$-probability equal to $1-\alpha$. This circular region corresponds to the Scheffé hyperbolic band over the whole line $(a, A)=(-\infty, \infty)$ which is therefore optimal. In Section 3, among all of the $1-\alpha$ level three-segment bands over a given finite interval $(a, A)$, the band that has the smallest region $R$ is identified to have a rhombus region $R$. In section 4 , among all of the $1-\alpha$ level two-segment bands, the band that has the smallest region $R$ corresponds to a square region $R$. Furthermore, the area of the square region $R$ that corresponds to the best $1-\alpha$ two-segment band is smaller than the area of the rhombus region that corresponds to the best $1-\alpha$ three-segment band. In Section 5 a comparison between the best three-segment band and the restricted hyperbolic band over a given finite interval $(a, A)$ is carried out. It is found that the restricted hyperbolic 
band is better than the best three-segment band if and only if the angle $\theta$ shown in Figure 1 is larger than a certain threshold. Section 6 compares the confidence bands considered in this article with respect to a specific data set. Section 7 has some concluding remarks, and the Appendix contains all of the proofs.

\section{The best band over the whole line}

The problem considered in this section is that of finding the region $R$ with the smallest area among all the regions in $R^{2}$ that have $\boldsymbol{T}$-probability equal to $1-\alpha$. That is to

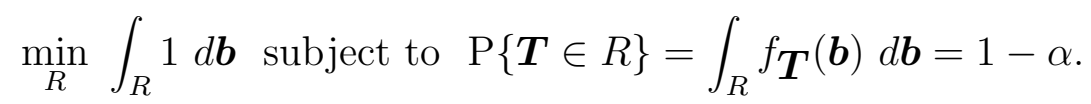

A bivariate $t$ random vector with covariance matrix $V$ and degrees of freedom $k$, denoted as $\boldsymbol{T}_{V, k}$, has a probability density function

$$
f_{T_{V, k}}(\boldsymbol{b})=\frac{1}{2 \pi \sqrt{|V|}}\left[1+\frac{1}{k} \boldsymbol{b}^{T} V^{-1} \boldsymbol{b}\right]^{-(2+k) / 2}, \quad \boldsymbol{b} \in R^{2}
$$

(see for example Tong (1990)). The random vector $\boldsymbol{T}$ has a covariance matrix $V$ equal to the identity matrix $I_{2}$ and so $\boldsymbol{T}=\boldsymbol{T}_{I_{2}, k}$.

The solution to the minimization problem (2.1) is given by

$$
R^{*}=\left\{\boldsymbol{b}: f_{\boldsymbol{T}}(\boldsymbol{b}) \geq d_{1}\right\}=\{\boldsymbol{b}:\|\boldsymbol{b}\| \leq r\}
$$

where the constants $d_{1}$ and $r$ are chosen so that the region $R^{*}$ has $\boldsymbol{T}$-probability equal to $1-\alpha$. This can be proved in a similar way as the Neyman-Pearson Lemma with the densities under the simple null and simple alternative hypotheses being replaced by $f_{\boldsymbol{T}^{(\cdot)}}$ and a constant function 1 respectively (see for example Lehman, 1986). The region $R^{*}$ is of the form of $R_{h}$ with $(a, A)=(-\infty, \infty)$ and so the corresponding confidence band is the hyperbolic band over the whole line $-\infty<x-\bar{x}<\infty$, i.e. the Scheffé band. Since $\|\boldsymbol{T}\|^{2} / 2$ has an $F$ distribution with 2 and $k$ degrees of freedom, $r$ is equal to $\sqrt{2 f_{2, k}^{\alpha}}$. 
In summary, using the MA criterion the Scheffé hyperbolic bands are best over the whole real line. However, in practice a regression model will not be used over the whole range of the covariate. Therefore, confidence bands only over a finite range, $x-\bar{x} \in(a, A)$ say, are of interest, and the restriction of the Scheffé band to $(a, A)$ has a confidence level greater than $1-\alpha$. The hyperbolic band over $(a, A)$ that has confidence level equal to $1-\alpha$ is considered in section 5 and it also has good properties under the MA criterion.

\section{The best three-segment band}

For given $X$ and $(a, A)$, there are two constants $c_{3,1}$ and $c_{3,2}$ in the three-segment band (1.3) to be determined to satisfy the confidence level requirement (1.1). Among the family of three-segment bands of form (1.3) and satisfying (1.1), the best band under the MA criterion minimizes the area of $R_{3}$. This minimization problem is equivalent to

$$
\max _{R_{3}} \int_{R_{3}} f_{\mathbf{T}}(\boldsymbol{t}) d \boldsymbol{t} \text { subject to area }\left(R_{3}\right)=\int_{R_{3}} 1 d \boldsymbol{t} \text { is fixed. }
$$

which is solved to find the best three-segment band in this section.

From Figure 1 , the angle $\theta \in(0, \pi)$ is formed by the vectors

$$
U \boldsymbol{a}=\left(\frac{1}{\sqrt{n}}, \frac{a}{\sqrt{s_{x x}}}\right)^{T} \text { and } U \boldsymbol{A}=\left(\frac{1}{\sqrt{n}}, \frac{A}{\sqrt{s_{x x}}}\right)^{T}
$$

and so

$$
\cos (\theta)=\left(\frac{1}{n}+\frac{a A}{s_{x x}}\right) / \sqrt{\left(\frac{1}{n}+\frac{a^{2}}{s_{x x}}\right)\left(\frac{1}{n}+\frac{A^{2}}{s_{x x}}\right)} .
$$

Notice that the angle $\theta$ depends only on the range of interest $(a, A)$ and the $X$ matrix.

When $X$ and $(a, A)$ are given, the angle $\theta$ of the parallelogram region $R_{3}$ is fixed and varying the critical constants $c_{3,1}$ and $c_{3,2}$ changes only the lengths of the two sides, $2 p$ and $2 q$ say, of the parallelogram $R_{3}$. Also, there is clearly a one-to-one correspondence between $(p, q)$ and $\left(c_{3,1}, c_{3,2}\right)$ for a given $\theta$. Since the area of the parallelogram is equal 
to $4 p q \sin \theta$, fixing the area of $R_{3}$ is equivalently to fixing the value of $p q$, which can be denoted by $c^{2}$. Furthermore, since the density function $f_{\mathbf{T}}(\boldsymbol{t})$ is invariant under an orthogonal transformation of the variables $\boldsymbol{t}$, it can be assumed that two sides of $R_{3}$ with length $2 q$ are parallel to one axis of the coordinate system.

Lemma 1 states that the $\boldsymbol{T}$-probability of the parallelogram $R_{3}$ is equal to the $\boldsymbol{T}_{V, k^{-}}$ probability of the rectangle $[-p, p] \times[-q, q]$ for a certain covariance matrix $V$. A proof is given in the Appendix.

Lemma 1. Under the notations above, we have

$$
\mathrm{P}\left\{\boldsymbol{T} \in R_{3}\right\}=\mathrm{P}\left\{\boldsymbol{N} /(\hat{\sigma} / \sigma) \in R_{3}\right\}=\mathrm{P}\left\{\boldsymbol{T}_{V, k} \in[-p, p] \times[-q, q]\right\}
$$

where the covariance matrix $V$ has the two diagonal elements equal to $1 / \sin ^{2} \theta$ and the two off-diagonal elements equal to $\cos \theta / \sin ^{2} \theta$.

The next result asserts that when the rectangle $[-p, p] \times[-q, q]$ approaches a square, given that the value of $p q$ is fixed at $c^{2}$, the probability $\mathrm{P}\left\{\boldsymbol{T}_{V, k} \in[-p, p] \times[-q, q]\right\}$ increases. A proof is given in the Appendix.

Lemma 2. Let $0<p \leq q$ and assume that $p q=c^{2}$ where $c$ is a fixed constant. Then, as a function of $p, \mathrm{P}\left\{\boldsymbol{T}_{V, k} \in[-p, p] \times[-q, q]\right\}=\mathrm{P}\left\{\boldsymbol{T}_{V, k} \in[-p, p] \times\left[-c^{2} / p, c^{2} / p\right]\right\}$ is strictly increasing over $p \in(0, c]$ and so attains its maximum at $p=q=c$.

Combining Lemmas 1 and 2 gives

Theorem 1. Among all three-segment bands of the form (1.3) and satisfying the confidence level requirement (1.1), the best one under the MA criterion is given uniquely by $c_{3,1}=c_{3,2}$. Its corresponding region $R_{3}$ is a rhombus.

Notice that the best three-segment band with $c_{3,1}=c_{3,2}$ is constructed so that the individual confidence level of the band at $a$ is equal to the individual confidence level of 
the band at $A$ (which would both be $\sqrt{1-\alpha}$ if $\sigma$ were known). Also, it is clear that this best three-segment band has the same width at $x-\bar{x}=a$ and $A$ (and hence the band has a constant width over $x-\bar{x} \in(a, A))$ if and only if $a=-A$.

\section{The best two-segment band and comparison with the best three-segment band}

A two-segment band corresponds to a rectangular region $R_{2}$. Changing the two critical values $\left(c_{2,1}, c_{2,2}\right)$ only changes the lengths of the two sides of $R_{2}$. Among all two-segment bands that satisfy the confidence level requirement (1.1), the best band under the MA criterion is identified by the following result which follows immediately from Lemma 2 for the special case of $\theta=\pi / 2$.

Theorem 2. Among all the two-segment bands of form (1.2) and satisfying the confidence level requirement (1.1), the best one under the MA criterion is given uniquely by $c_{2,1}=c_{2,2}$. Its corresponding region $R_{2}$ is a square.

Notice that this best two-segment band is constructed with the individual confidence intervals for $b_{0}$ and $b_{1}$ having equal confidence levels (which would both be $\sqrt{1-\alpha}$ if $\sigma$ were known).

Now we compare the best two-segment band with the best three-segment band. That is, if $R_{2}$ is a square and $R_{3}$ is a rhombus and both have the $\boldsymbol{T}$-probability equal to $1-\alpha$, then which region, $R_{2}$ or $R_{3}$, has a smaller area? The answer is $R_{2}$ as asserted in Theorem 3 below. The proof hinges on the following lemma, whose proof is given in the Appendix.

Lemma 3. Let $R_{3}$ be a rhombus centered at the origin and the line segments that connect the opposite vertices have lengths $2 x$ and $2 c^{2} / x$ respectively as illustrated in Figure 
2, where $c^{2}$ is a fixed constant. Then, as a function of $x, \mathrm{P}\left\{\boldsymbol{T} \in R_{3}\right\}$ is increasing over $x \in(0, c)$.

From Lemma 3 it is clear that, among all the rhombuses that are centered at the origin and that have the same area, the square has the largest $\boldsymbol{T}$-probability. Equivalently, among all the rhombuses that are centered at the origin and that have the same $\boldsymbol{T}$-probability, the square has the smallest area. This implies

Theorem 3. The square region $R_{2}$ that corresponds to the best $1-\alpha$ level two-segment band has a smaller area than the rhombus region $R_{3}$ that corresponds to the best $1-\alpha$ level three-segment confidence band.

Consequently, under the MA criterion the best two-segment band is better than the best three-segment band. Note, however, a two-segment band is intrinsically over the whole line, that is, a restriction of an exact $1-\alpha$ level two-segment band to any finite interval has a confidence level greater than $1-\alpha$. So if a confidence band over a finite interval of the covariate is required then a two-segment band can always be improved upon and hence should not be used. On the other hand, a restriction of an exact $1-\alpha$ level three-segment band to any interval that contains $(a, A)$ still has a confidence level equal to $1-\alpha$.

\section{Comparison between the best three-segment band and the hyperbolic band over a finite interval $(a, A)$}

If attention is restricted to an interval $(a, A)$, then confidence bands with an exact confidence level of $1-\alpha$ over this interval can be formed with a hyperbolic shape or as a three-segment bands. These two options are compared in this section with respect to the 
MA criterion.

From Section 3, the region $R_{3}$ that corresponds to the best three-segment band is given by a rhombus. The region $R_{h}$ that corresponds to a hyperbolic band over $(a, A)$ is given by a spindle. Since the density function of $\boldsymbol{T}$ is invariant under an orthogonal transformation, the rhombus and the spindle can be positioned as in Figure 3. If both of these two regions have the $\boldsymbol{T}$-probability equal to $1-\alpha$ then which region, $R_{3}$ or $R_{h}$, has a smaller area? The answer to this question is not definitive but depends on the magnitude of the angle $\theta$ shown in Figures 1 and 3, which can be calculated from (3.1).

For given $k, \alpha$ and $\theta$, the values of $r_{h}=r_{h}(\theta ; k, \alpha)$ and $r_{3}=r_{3}(\theta ; k, \alpha)$ shown in Figure 3 are uniquely determined to satisfy the $1-\alpha$ confidence level requirement (1.1), i.e.

$$
\int_{R_{h}} f_{\boldsymbol{T}}(\boldsymbol{b}) d \boldsymbol{b}=1-\alpha \text { and } \int_{R_{3}} f_{\boldsymbol{T}^{(}}(\boldsymbol{b}) d \boldsymbol{b}=1-\alpha
$$

By using the method of Wynn and Bloomfield (1971), we have

$$
\int_{R_{h}} f_{\boldsymbol{T}}(\boldsymbol{b}) d \boldsymbol{b}=1-\frac{\theta}{\pi}\left(1+\frac{r_{h}^{2}}{k}\right)^{-k / 2}-\frac{2}{\pi} \int_{0}^{(\pi-\theta) / 2}\left[1+\frac{r_{h}^{2}}{k \sin ^{2}(\theta / 2+\beta)}\right]^{-k / 2} d \beta .
$$

In particular, when $k=\infty$ we have

$$
\int_{R_{h}} f_{\boldsymbol{T}}(\boldsymbol{b}) d \boldsymbol{b}=1-\frac{\theta}{\pi} \exp \left(-\frac{r_{h}^{2}}{2}\right)-\frac{2}{\pi} \int_{0}^{(\pi-\theta) / 2} \exp \left[-\frac{r_{h}^{2}}{2 \sin ^{2}(\theta / 2+\beta)}\right] d \beta .
$$

Similarly, for the best three-segment band, we have

$$
\int_{R_{3}} f_{\boldsymbol{T}}(\boldsymbol{b}) d \boldsymbol{b}=1-\frac{2}{\pi} \int_{0}^{\pi / 2}\left[1+\frac{r_{3}^{2}}{k \sin ^{2}(\theta / 2+\beta)}\right]^{-k / 2} d \beta
$$

and, when $k=\infty$,

$$
\int_{R_{3}} f_{\boldsymbol{T}}(\boldsymbol{b}) d \boldsymbol{b}=1-\frac{2}{\pi} \int_{0}^{\pi / 2} \exp \left[-\frac{r_{3}^{2}}{2 \sin ^{2}(\theta / 2+\beta)}\right] d \beta
$$

After solving $r_{h}$ and $r_{3}$ from (5.1) numerically by using the expressions above, the area of $R_{h}$ is readily calculated to be given by

$$
\operatorname{area}\left(R_{h}\right)=r_{h}^{2}[\theta+2 \cot (\theta / 2)]
$$


and the area of $R_{3}$ is given by

$$
\operatorname{area}\left(R_{3}\right)=\frac{4 r_{3}^{2}}{\sin (\theta)}
$$

Hence

$$
e(\theta):=\frac{\operatorname{area}\left(R_{3}\right)}{\operatorname{area}\left(R_{h}\right)}=\frac{4 r_{3}^{2}}{r_{h}^{2}[\theta+2 \cot (\theta / 2)] \sin (\theta)}
$$

is of interest in order to compare the areas of $R_{h}$ and $R_{3}$, and it can be regarded as the relative efficiency of the hyperbolic band to the best three-segment band under the MA criterion.

\begin{tabular}{|c|c|c|c|c|c|c|c|c|c|}
\hline & $e_{l}$ & $\theta^{*}$ & $e(3.10)$ & $e_{l}$ & $\theta^{*}$ & $e(3.10)$ & $e_{l}$ & $\theta^{*}$ & $e(3.10)$ \\
\hline & $\alpha=0.10$ & & & $\alpha=0.05$ & & & $\alpha=0.01$ & & \\
\hline$k=6$ & 0.986 & 1.484 & 17.07 & 0.985 & 1.473 & 18.17 & 0.984 & 1.451 & 19.58 \\
\hline$k=10$ & 0.985 & 1.485 & 17.57 & 0.984 & 1.469 & 18.87 & 0.983 & 1.444 & 20.64 \\
\hline$k=20$ & 0.985 & 1.487 & 17.95 & 0.983 & 1.469 & 19.41 & 0.982 & 1.434 & 21.48 \\
\hline$k=\infty$ & 0.984 & 1.489 & 18.35 & 0.983 & 1.468 & 19.96 & 0.982 & 1.420 & 22.33 \\
\hline
\end{tabular}

Table 1. The values of $e_{l}, \theta^{*}$ and $e(3.10)$

Computations have been made of $e(\theta), \theta \in(0, \pi)$ for all combinations of $\alpha=0.01,0.05,0.10$ and $k=6,10,20, \infty$. The following pattern of the function $e(\theta)$ is observed for all the combinations of $\alpha$ and $k$. The function $e(\theta)$ first decreases and then increases over $\theta \in(0, \pi)$. When $\theta$ approaches 0 from the right, $e(\theta)$ approaches one. At a certain threshold value $\theta^{*}, e\left(\theta^{*}\right)$ is equal to one. When $\theta$ is large (close to $\left.\pi\right)$, the value of $e(\theta)$ becomes very large. Figure 4 provides a plot of $e(\theta)$ for $\alpha=0.05$ and $k=10$, the shape of which is typical for all combinations of $\alpha$ and $k$. The diamond on the curve identifies the point at which $e(\theta)=1$. Table 1 gives $e_{l}=\min _{(0, \pi)} e(\theta), \theta^{*}$ at which $e\left(\theta^{*}\right)=1$, and $e(3.10)$ which provides some indication on how large $e(\theta)$ can be when $\theta$ approaches $\pi$ from the left. 
The following conclusions can be drawn. When $\theta<\theta^{*}$ the best three-segment band is better than the hyperbolic band under the MA criterion, and when $\theta>\theta^{*}$ the hyperbolic band is better than the best three-segment band. From Table 1 the value of $\theta^{*}$ is between 1.4 and 1.5 , which is slightly smaller than $\pi / 2$. So one could calculate the angle $\theta$ from (3.1) and hence judge whether to use the best three-segment band or the hyperbolic band. For the special case of $a=-A$, a larger value of $n a^{2} / s_{x x}$ gives a larger angle $\theta$ and so the hyperbolic band is preferable to the best three-segment band.

However, notice from Table 1 that the minimum value of $e(\theta), e_{l}$, is pretty close to one (while the smallest $e_{l}$ is 0.982 ). This indicates that the loss of efficiency due to the use of the hyperbolic band instead of the best three-segment band is rather small. On the other hand the potential loss of efficiency due to the use of the best three-segment band rather than the hyperbolic band can be huge, especially when $\theta$ is close to $\pi$. Therefore, it is reasonable to always recommend that the hyperbolic band be employed.

\section{An Example}

Kleinbaum et al. (1998, pp.192) provide a data set on how systolic blood pressure (SBP) changes with age for a group of forty males. The data points and the fitted regression line which is $S B P=155.146+0.961(a g e-a \bar{g} e)$ are plotted in Figure 5. Also $\bar{x}=46.92$, the minimum age $\min \left(x_{i}\right)=18$, the maximum age $\max \left(x_{i}\right)=70, s_{x x}=8623.5591$, and $\hat{\sigma}=8.479$ with 38 degrees of freedom.

A simultaneous confidence band can be used to quantify the plausible range of the true regression line. Several simultaneous confidence bands can be constructed as discussed in the introduction, and the question is 'which band should be used?' Under the MA criterion introduced in this paper, a better band should have a smaller area of the confidence region and so contain fewer plausible candidates for the unknown regression line. 
Since $\bar{x}=46.92, \min \left(x_{i}\right)=18$ and $\max \left(x_{i}\right)=70$, it is sensible to construct a simultaneous confidence band over $x-\bar{x} \in(18-46.92,70-46.92)=(-28.92,23.08)=(a, A)$. In this case the angle $\theta$ is calculated from (3.1) to be $\theta=2.105$. For $\alpha=0.05$, one can calculate the critical value of the hyperbolic band over $(-28.92,23.08)$ from the first equation in $(5.1)$, by using the expression $(5.2)$, to get $r_{h}=2.514$. The area of the region $R_{h}$ corresponding to this confidence band is then calculated from (5.4) to be area $\left(R_{h}\right)=20.512$.

If one is interested in a three-segment confidence band over the interval $(a, A)=$ $(-28.92,23.08)$, then the critical constant of the best three-segment band under the MA criterion, $r_{3}$, is calculated from the second equation in (5.1), by using the expression (5.3), to get $r_{3}=2.296$. The area of the region $R_{3}$ corresponding to this best threesegment confidence band is then calculated from (5.5) to be area $\left(R_{3}\right)=24.501$. So, for this particular example, the best three-segment band over $(-28.92,23.08)$ contains $(24.501-20.512) / 20.512=19 \%$ more plausible candidates of the true regression line than the hyperbolic band over $(-28.92,23.08)$, and hence the hyperbolic band is better than the best three-segment band. This is expected from the observations made in Section 5 since $\theta=2.105$ is much larger than $\theta=1.5$. Both the hyperbolic and best three-segment bands are plotted in Figure 5. The best three-segment band is identified in the picture. The hyperbolic band is given by the pair of solid curves over the interval $(-28.92,23.08)$. It is so close to the Scheffé hyperbolic band over the whole range $(-\infty, \infty)$ that the two bands cannot be distinguished over $(-28.92,23.08)$ in the picture.

From Theorem 3, the best two-segment band is always better than the best threesegment band under the MA criterion. Indeed, from Theorem 2, the critical constants $c_{2,1}=c_{2,2}=r_{2}$ of the best two-segment band is equal to $r_{3}$ but with $\theta=\pi / 2$, while the area of the region $R_{2}$ corresponding to this best two-segment band is clearly equal to $4 r_{2}^{2}$. So with $r_{2}=2.326$ it follows that $\operatorname{area}\left(R_{2}\right)=21.085$ so that $\operatorname{area}\left(R_{2}\right)<\operatorname{area}\left(R_{3}\right)$ as 
expected. Note however that area $\left(R_{2}\right)>\operatorname{area}\left(R_{h}\right)$. The best two-segment band is also plotted in Figure 5.

From Section 2, the best confidence band under the MA criterion over the whole real line is the Scheffé hyperbolic band. Its critical value is $c_{h}=\sqrt{2 f_{2,38}^{0.05}}=2.547$ and the area of the corresponding $R_{h}$ is $\operatorname{area}\left(R_{h}\right)=\pi c_{h}^{2}=20.380$, which is smaller, but only marginally, than that of the hyperbolic band over $(-28.92,23.08)$. This band is given in Figure 5 by the pair of solid curves over $(-\infty, \infty)$, which is undistinguishable from the hyperbolic band over $(-28.92,23.08)$. The confidence level of the Scheffé hyperbolic band over the restricted region $(a, A)=(-28.92,23.08)$ is 0.955 .

Figure 6 depicts the $R$-regions corresponding to the best three-segment band (given by the rhombus), the best two-segment band (given by the square), the hyperbolic band over $(-28.92,23.08)$ (given by the spindle), and the Scheffé band (given by the circle).

Finally, both the two-segment and Scheffé bands are over the whole predictor variable range $(-\infty, \infty)$. The Scheffé band derives constraints on the regression coefficients $\boldsymbol{b}$ by bounding the regression line at every $x \in(-\infty, \infty)$. This is not sensible from a practical point of view since the regression model in this example is clearly wrong when $x=a g e$ is too small or too large. Indeed any extrapolation of a regression model outside the observed range of the covariate may be risky as argued frequently in the statistics literature. The restriction of the Scheffé band to the observed range $(-28.92,23.08)$ is not efficient since it is dominated by the hyperbolic band over $(-28.92,23.08)$ which used $r_{h}=2.514$. The ratio of the areas of the $R$-regions corresponding to the restriction of the Scheffé band (which uses critical value 2.547$)$ and the hyperbolic band over $(-28.92,23.08)$ is $(2.547 / 2.514)^{2}=1.03$.

Similarly, the restriction of the two-segment band to $(-28.92,23.08)$ is not efficient either since this restriction can be shrunk in same proportion over $(-28.92,23.08)$ to result in a (four-segment) band which has confidence level equal to $95 \%$ and dominates the re- 
striction of the two-segment band. In conclusion, the hyperbolic band over $(-28.92,23.08)$ is preferable to the other bands under the MA criterion for this particular example.

\section{Conclusions}

A new optimality criterion for two-sided confidence band construction for simple linear regression models is proposed in this article. Under this criterion, the Scheffé band which is over the whole line is the best among all the $1-\alpha$ level confidence bands over the whole real line. The best two-segment and the best three-segment bands are also identified. Furthermore, it is shown that the best two-segment band is always better than the best three-segment band under this criterion.

In most real problems, a regression model holds only over a finite interval of the covariate and so a confidence band over a finite interval is of interest. In this case, the hyperbolic band with an exact confidence level over the restricted region can also be recommended, although strictly speaking it does not dominate the best three-segment band. Which of these two bands is better depends on the angle $\theta$, and when $\theta$ is large the hyperbolic band is better, while when $\theta$ is small the best three-segment band is better. However, the hyperbolic band can only be very slightly worse than the best three-segment band.

In a recent paper, Efron (2006) considered the construction of minimum volume confidence regions for a normal mean vector. For three or more than three normal means, where 'anomalies' (such as Stein's paradox) usually occur, the standard ellipsoidal confidence region can be improved upon in terms of the volume of a confidence region. Our paper however focuses on a simple linear regression model, which involves only two normal means. Also each of the improved confidence regions given in Efron (2006) uses acceptance sets of different shapes for different mean vectors. The formulation of the confidence band problem in our paper is such (see Section 1.3) that all the confidence sets for $\boldsymbol{b}$ consid- 
ered have the form $C=\left\{\mathbf{b}: U^{-1}(\boldsymbol{b}-\hat{\boldsymbol{b}}) / \hat{\sigma} \in R\right\}$, and so all the acceptance sets of each confidence set are of the same shape.

\section{Acknowledgements}

We would like to thank the Editor, Associate Editor and referees for several critical and constructive comments.

\section{References}

Al-Saidy, O.M., Piegorsch, W.W., West, R.W. and Nitcheva, D.K. (2003), "Confidence bands for low-dose risk estimation with quantal response data," Biometrics, 59, 1056-1062. Atkinson, A.C. and Donev, A.N. (1992), "Optimum Experimental Designs," Oxford University Press.

Bowden, D. C. and Graybill, F. A. (1966), "Confidence bands of uniform and proportional width for linear models," Journal of the American Statistical Association, 61, 182-198.

Bowden, D.C. (1970), "Simultaneous confidence bands for linear regression models," Journal of the American Statistical Association, 65, 413-421.

Efron, B. (2006), "Minimum volume confidence region for a multivariate normal mean vector," Journal of the Royal Statistical Society (B), 68, 655-670.

Gafarian, A. V. (1964), "Confidence bands in straight line regression," Journal of the American Statistical Association, 59, 182-213.

Graybill, F. A. and Bowden, D. C. (1967), "Linear Segment Confidence Bands for Simple Linear Regression Models," Journal of the American Statistical Association, 62, 403-408.

Khorasani, F and Milliken, G.A. (1979), "On the exactness of confidence bands about a linear model," Journal of the American Statistical Association, 74, 446-448.

Kleinbaum, D.G., Kupper, L.L., Muller, K.E. and Nizam, A. (1998), “Applied Regression Analysis and Other Multivariable Methods," 3rd Edition, Duxbury Press.

Lehmann, E.L. (1986), “ Testing Statistical Hypotheses," 2nd edition, Wiley. 
Liu, W., Jamshidian, M., Zhang, Y. (2004), "Multiple comparison of several regression models," Journal of the American Statistical Association, 99, 395-403.

Naiman, D.Q. (1983), “Comparing Scheffé-type to constant-width confidence bands in regression," Journal of the American Statistical Association, 78, 906-912.

Naiman, D.Q. (1984), "Average width optimality of simultaneous confidence bands," $A n$ nals of Statistics, 12, 1199-1214.

Naiman, D.Q. (1987), “Minimax regret simultaneous confidence bands for multiple regression functions," Journal of the American Statistical Association, 82, 894-901.

Piegorsch, W.W. (1985a), "Average-width optimality for confidence bands in simple linear regression," Journal of the American Statistical Association, 80, 692-697.

Piegorsch, W.W. (1985b), "Admissible and optimal confidence bands in simple linear regression," Annals of Statistics, 13, 801-810.

Piegorsch, W.W. (1987), "On confidence bands and set estimators for the simple linear model," Statistics $\&$ Probability Letters, 5(6), 409-413.

Piegorsch, W.W., West R.W., Al-Saidy O.M. and Bradley, K.D. (2000), "Asymmetric confidence bands for simple linear regression over bounded intervals," Computational Statistics E) Data Analysis, 34(2), 193-217.

Piegorsch, W.W., West R.W., Pan, W. and Kodell, R. (2005), "Low dose risk estimation via simultaneous statistical inferences," Journal of the Royal Statistical Society (C), 245258.

Pukelsheim, F., "Optimal Design of Experiments," Wiley.

Scheffe, H. (1953), "A method for judging all contrasts in the analysis of variance," Biometrika, 41, 153-169.

Scheffe, H. (1959), "Analysis of Variance," Wiley.

Spurrier, J.D. (1999), "Exact Confidence Bounds for All Contrasts of Three or More Re- 
gression Lines," Journal of the American Statistical Association, 94, 483-88.

Tong, Y.L. (1990), "Multivariate Normal Distribution," Springer-Verlag.

Uusipaikka, E. (1983), "Exact confidence bands for linear-regression over intervals," Journal of the American Statistical Association, 78, 638-644.

Working, H. and Hotelling, H. (1929), "Applications of the theory of error to the interpretation of trends," Journal of the American Statistical Association, 24, 73-85.

Wynn, H.P. and Bloomfield, P. (1971), "Simultaneous confidence bands in regression analysis," Journal of the Royal Statistical Society (B), 33, 202-217.

\section{Appendix}

Let $\phi$ and $\Phi$ be the density and cumulative probability functions of the standard normal distribution respectively. Let $\boldsymbol{N}_{V}$ be a bivariate normal random vector with zero mean vector, covariance matrix $V$, and independent of $\hat{\sigma} / \sigma$. The joint pdf of $\boldsymbol{N}_{V}$ is denoted by $f_{\boldsymbol{N}_{V}}(\cdot, \cdot)$. We firstly outline a

Proof of Lemma 1. By conditioning on $\hat{\sigma} / \sigma$, it suffices to prove the result for the special case of $k=\infty$, i.e. when $\sigma$ is known and so $\hat{\sigma} / \sigma=1$. Now positioning $R_{3}$ so that its two sides of length $2 p$ are parallel to the $x_{1}$-axis and the other two sides of length $2 q$ form angle $\theta$ with the $x_{1}$-axis. Hence

$$
\mathrm{P}\left\{\boldsymbol{N} \in R_{3}\right\}=\int_{R_{3}} f_{\boldsymbol{N}}(\boldsymbol{x}) d \boldsymbol{x}=\int_{-q \sin \theta}^{q \sin \theta} \int_{x_{2} \cot \theta-p}^{x_{2} \cot \theta+p} \phi\left(x_{1}\right) \phi\left(x_{2}\right) d x_{1} d x_{2}
$$

Changing variables $x_{1}=-u+v \cos \theta$ and $x_{2}=v \sin \theta$ gives the double integral above equal to

$$
\int_{-q}^{q} \int_{-p}^{p} f_{\boldsymbol{N}_{V}}(u, v) d u d v=\mathrm{P}\left\{\boldsymbol{N}_{V} \in[-p, p] \times[-q, q]\right\}
$$

where $V$ has its two diagonal elements equal to $1 / \sin ^{2} \theta$ and two off-diagonal elements equal to $\cos \theta / \sin ^{2} \theta$. The proof is thus complete. 
The next result is required in the proof of Lemma 2 and can be proved by direct manipulation.

Lemma A1. For $a>0$ and $b, c \in R^{1}$, we have

$$
\int_{-\infty}^{\infty} \frac{1}{a} \phi\left(\frac{x}{a}\right) \phi(x+b) \Phi(x+c) d x=\exp \left[\frac{-b^{2}}{2\left(1+a^{2}\right)}\right] \Phi\left[\sqrt{\frac{1+a^{2}}{1+2 a^{2}}}\left(c-\frac{b a^{2}}{1+a^{2}}\right)\right] / \sqrt{2 \pi\left(1+a^{2}\right)}
$$

Next we give a

Proof of Lemma 2. Express $\boldsymbol{T}_{V, k}$ as $\boldsymbol{T}_{V, k}=\boldsymbol{N}_{V} /(\hat{\sigma} / \sigma)$. By conditioning on the random variable $\hat{\sigma} / \sigma$, it is sufficient to show that $\mathrm{P}\left\{\boldsymbol{N}_{V} / s \in[-p, p] \times[-q, q]\right\}$ is strictly increasing over $p \in(0, c)$ for each given value of $\hat{\sigma} / \sigma=s \in(0, \infty)$. Note further that

$$
\begin{aligned}
\mathrm{P}\left\{\frac{\boldsymbol{N}_{V}}{s} \in[-p, p] \times[-q, q]\right\} & =\mathrm{P}\left\{\boldsymbol{N}_{V} \sin \theta \in[-p s \sin \theta, p s \sin \theta] \times[-q s \sin \theta, q s \sin \theta]\right\} \\
& =\mathrm{P}\left\{\boldsymbol{N}_{V_{0}} \in[-p s \sin \theta, p s \sin \theta] \times[-q s \sin \theta, q s \sin \theta]\right\}
\end{aligned}
$$

where $V_{0}=\left(\begin{array}{cc}1 & \rho \\ & \\ \rho & 1\end{array}\right)$ with $\rho=\cos \theta$. So it suffices to show that this probability is strictly increasing in $p s \sin \theta \in(0, c s \sin \theta)$. Equivalently, it is sufficient to prove the lemma for the special case of $k=\infty$ and $V=V_{0}$. A proof of this is given next.

Let $\lambda=c-p \geq 0$. We shall show that

$D(\lambda):=\mathrm{P}\left\{\boldsymbol{N}_{V_{0}} \in[-c, c] \times[-c, c]\right\}-\mathrm{P}\left\{\boldsymbol{N}_{V_{0}} \in[-(c-\lambda), c-\lambda] \times\left[-c^{2} /(c-\lambda), c^{2} /(c-\lambda)\right]\right\}$ is strictly increasing, i.e. $D^{\prime}(\lambda)>0$, over $\lambda \in(0, c)$ which suffices. Note that $D(\lambda)=$ $4\left[v_{1}(\lambda)-v_{2}(\lambda)\right]$ where

$$
v_{1}(\lambda)=\mathrm{P}\left\{\boldsymbol{N}_{V_{0}} \in[c-\lambda, c] \times[0, c]\right\} \text { and } v_{2}(\lambda)=\mathrm{P}\left\{\boldsymbol{N}_{V_{0}} \in\left[c, c^{2} /(c-\lambda)\right] \times[0, c-\lambda]\right\} .
$$

Represent the two components of $\boldsymbol{N}_{V_{0}}$ as $N_{1}=\sqrt{1-\rho} W_{1}+\sqrt{\rho} W_{0}$ and $N_{2}=\sqrt{1-\rho} W_{2}+$ $\sqrt{\rho} W_{0}$ where $W_{0}, W_{1}, W_{2}$ are i.i.d. standard normal random variables. Then $v_{1}(\lambda)=\mathrm{P}\left\{c-\lambda \leq \sqrt{1-\rho} W_{1}+\sqrt{\rho} W_{0} \leq c, 0 \leq \sqrt{1-\rho} W_{2}+\sqrt{\rho} W_{0} \leq c\right\}$ 


$$
\begin{aligned}
& =\int_{-\infty}^{\infty} \phi(x)\left[\Phi\left(\frac{c-\sqrt{\rho} x}{\sqrt{1-\rho}}\right)-\Phi\left(\frac{c-\lambda-\sqrt{\rho} x}{\sqrt{1-\rho}}\right)\right]\left[\Phi\left(\frac{c-\sqrt{\rho} x}{\sqrt{1-\rho}}\right)-\Phi\left(\frac{-\sqrt{\rho} x}{\sqrt{1-\rho}}\right)\right] d x \text { and } \\
v_{2}(\lambda) & =\mathrm{P}\left\{c \leq \sqrt{1-\rho} W_{1}+\sqrt{\rho} W_{0} \leq c^{2} /(c-\lambda), 0 \leq \sqrt{1-\rho} W_{2}+\sqrt{\rho} W_{0} \leq c-\lambda\right\} \\
& =\int_{-\infty}^{\infty} \phi(x)\left[\Phi\left(\frac{c^{2} /(c-\lambda)-\sqrt{\rho} x}{\sqrt{1-\rho}}\right)-\Phi\left(\frac{c-\sqrt{\rho} x}{\sqrt{1-\rho}}\right)\right]\left[\Phi\left(\frac{c-\lambda-\sqrt{\rho} x}{\sqrt{1-\rho}}\right)-\Phi\left(\frac{-\sqrt{\rho} x}{\sqrt{1-\rho}}\right)\right] d x .
\end{aligned}
$$

Now direct calculation shows that $v_{1}^{\prime}(\lambda)-v_{2}^{\prime}(\lambda)=g_{1}-g_{2}$ where

$$
\begin{aligned}
& g_{1}=\frac{1}{\sqrt{1-\rho}} \int_{-\infty}^{\infty} \phi(x) \phi\left(\frac{\sqrt{\rho} x-c+\lambda}{\sqrt{1-\rho}}\right)\left[\Phi\left(\frac{c^{2} /(c-\lambda)-\sqrt{\rho} x}{\sqrt{1-\rho}}\right)-\Phi\left(\frac{-\sqrt{\rho} x}{\sqrt{1-\rho}}\right)\right] d x \text { and } \\
& g_{2}=\frac{c^{2}}{\sqrt{\rho}(c-\lambda)^{2}} \int_{-\infty}^{\infty} \phi\left(\frac{\sqrt{(1-\rho)} x}{\sqrt{\rho}}\right) \phi\left(x-\frac{c^{2}}{(c-\lambda) \sqrt{1-\rho}}\right)\left[\Phi(x)-\Phi\left(x-\frac{c-\lambda}{\sqrt{1-\rho}}\right)\right] d x .
\end{aligned}
$$

It follows from some changes of variables and Lemma A1 that

$$
\begin{aligned}
g_{1} & =\frac{1}{\sqrt{2 \pi}} \exp \left[-\frac{(c-\lambda)^{2}}{2}\right]\left[\Phi\left(\frac{\rho(c-\lambda)}{\sqrt{1-\rho^{2}}}\right)-\Phi\left(\frac{\rho(c-\lambda)}{\sqrt{1-\rho^{2}}}-\frac{c^{2}}{(c-\lambda) \sqrt{1-\rho^{2}}}\right)\right] \\
& =\frac{c}{(c-\lambda) \sqrt{2 \pi}} \exp \left[-\frac{(c-\lambda)^{2}}{2}\right] \int_{0}^{c / \sqrt{1-\rho^{2}}} \phi\left(\frac{c x}{c-\lambda}-\frac{\rho(c-\lambda)}{\sqrt{1-\rho^{2}}}\right) d x \text { and } \\
g_{2} & =\frac{c^{2}}{(c-\lambda)^{2} \sqrt{2 \pi}} \exp \left[-\frac{c^{4}}{2(c-\lambda)^{2}}\right]\left[\Phi\left(\frac{\rho c^{2}}{(c-\lambda) \sqrt{1-\rho^{2}}}\right)-\Phi\left(\frac{\rho c^{2}}{(c-\lambda) \sqrt{1-\rho^{2}}}-\frac{(c-\lambda)}{\sqrt{1-\rho^{2}}}\right)\right] \\
& =\frac{c}{(c-\lambda) \sqrt{2 \pi}} \exp \left[-\frac{c^{4}}{(c-\lambda)^{2}}\right] \int_{0}^{c / \sqrt{1-\rho^{2}}} \phi\left(\frac{(c-\lambda) x}{c}-\frac{\rho c^{2}}{(c-\lambda) \sqrt{1-\rho^{2}}}\right) d x .
\end{aligned}
$$

So

$$
D^{\prime}(\lambda)=4\left[v_{1}^{\prime}(\lambda)-v_{2}^{\prime}(\lambda)\right]=4\left(g_{1}-g_{2}\right)=\frac{4 c}{(c-\lambda) \sqrt{2 \pi}} \int_{0}^{c / \sqrt{1-\rho^{2}}} h(x) d x
$$

where

$$
h(x)=\exp \left[-\frac{(c-\lambda)^{2}}{2}\right] \phi\left(\frac{c x}{c-\lambda}-\frac{\rho(c-\lambda)}{\sqrt{1-\rho^{2}}}\right)-\exp \left[-\frac{c^{4}}{(c-\lambda)^{2}}\right] \phi\left(\frac{(c-\lambda) x}{c}-\frac{\rho c^{2}}{(c-\lambda) \sqrt{1-\rho^{2}}}\right) .
$$

It is now straightforward to shown that $h(x)$ is positive over $x \in\left(0, c / \sqrt{1-\rho^{2}}\right)$ for $\lambda \in$ $(0, c)$. We have thus shown that $D^{\prime}(\lambda)>0$ for $\lambda \in(0, c)$ as required. The proof is thus complete.

Next we outline a

Proof of Lemma 3. By conditioning on $\hat{\sigma} / \sigma$ as before it suffices to show that $\mathrm{P}\{\boldsymbol{N} \in$ $\left.R_{3}\right\}$ is increasing in $x \in(0, c)$. Let $R_{2}$ be a square positioned as in Figure 2 and having 
area equal to $2 c^{2}$. It suffices to show that

$$
g(x):=\mathrm{P}\left\{\boldsymbol{N} \in R_{3}\right\}-P\left\{\boldsymbol{N} \in R_{2}\right\}
$$

is increasing in $x \in(0, c)$. Note that $g(x)=4\left[\mathrm{P}\left\{\boldsymbol{N} \in S_{1}\right\}-\mathrm{P}\left\{\boldsymbol{N} \in S_{2}\right\}\right]$ where the regions $S_{1}$ and $S_{2}$ are depicted in Figure 2. Now

$$
\begin{aligned}
u_{1}(x) & :=\mathrm{P}\left\{\boldsymbol{N} \in S_{1}\right\}=\int_{0}^{c x /(c+x)} d z_{2} \int_{c\left(1-z_{2} / c\right)}^{c^{2}\left(1-z_{2} / x\right) / x} \phi\left(z_{1}\right) \phi\left(z_{2}\right) d z_{1} \\
& =\int_{0}^{c x /(c+x)} \phi(z)\left\{\Phi\left[c^{2}(1-z / x) / x\right]-\Phi[c(1-z / c)]\right\} d z
\end{aligned}
$$

and so

$$
\begin{aligned}
u_{1}^{\prime}(x) & =-\int_{0}^{c x /(c+x)} \phi(z) \phi\left[c^{2}(x-z) / x^{2}\right] c^{2}(1-2 z / x) / x^{2} d z \\
& =-\left\{\int_{0}^{x / 2}+\int_{x / 2}^{c x /(c+x)}\right\} \phi(z) \phi\left[c^{2}(x-z) / x^{2}\right] c^{2}(1-2 z / x) / x^{2} d z .
\end{aligned}
$$

Similarly,

$$
u_{2}(x):=\mathrm{P}\left\{\boldsymbol{N} \in S_{2}\right\}=\int_{0}^{c^{2} /(c+x)} \phi(z)\left\{\Phi[c(1-z / c) / x]-\Phi\left[x\left(1-x z / c^{2}\right)\right]\right\} d z
$$

and

$$
\begin{aligned}
u_{2}^{\prime}(x) & =-\int_{0}^{c^{2} /(c+x)} \phi(z) \phi\left[x\left(1-x z / c^{2}\right)\right]\left(1-2 x z / c^{2}\right) d z \\
& =-\int_{0}^{x^{2} /(c+x)} \phi\left(c^{2} z / x^{2}\right) \phi(x-z)(1-2 z / x) c^{2} / x^{2} d z \\
& =-\left\{\int_{0}^{x / 2}-\int_{x^{2} /(c+x)}^{x / 2}\right\} \phi\left(c^{2} z / x^{2}\right) \phi(x-z)(1-2 z / x) c^{2} / x^{2} d z
\end{aligned}
$$

Now it is easy to show, after some changes of variables, that

$$
\int_{x / 2}^{c x /(c+x)} \phi(z) \phi\left[c^{2}(x-z) / x^{2}\right] c^{2}(1-2 z / x) / x^{2} d z+\int_{x^{2} /(c+x)}^{x / 2} \phi\left(c^{2} z / x^{2}\right) \phi(x-z)(1-2 z / x) c^{2} / x^{2} d z=0 .
$$

Hence

$$
g^{\prime}(x)=4\left[u_{1}^{\prime}(x)-u_{2}^{\prime}(x)\right]=4 \int_{0}^{x / 2} J(x)(1-2 z / x) c^{2} / x^{2} d z
$$

where $J(z)=\phi\left(c^{2} z / x^{2}\right) \phi(x-z)-\phi(z) \phi\left[c^{2}(x-z) / x^{2}\right]$. It is straight forward to show that $J(z)>0$ for $z \in(0, x / 2)$ and $x \in(0, c)$, and so $g^{\prime}(x)>0$ for $x \in(0, c)$ as required. The proof is thus complete. 
Figure 1: $R_{h}$ is given by the spindle, $R_{2}$ is given by the rectangle, and $R_{3}$ is given by the parallelogram.

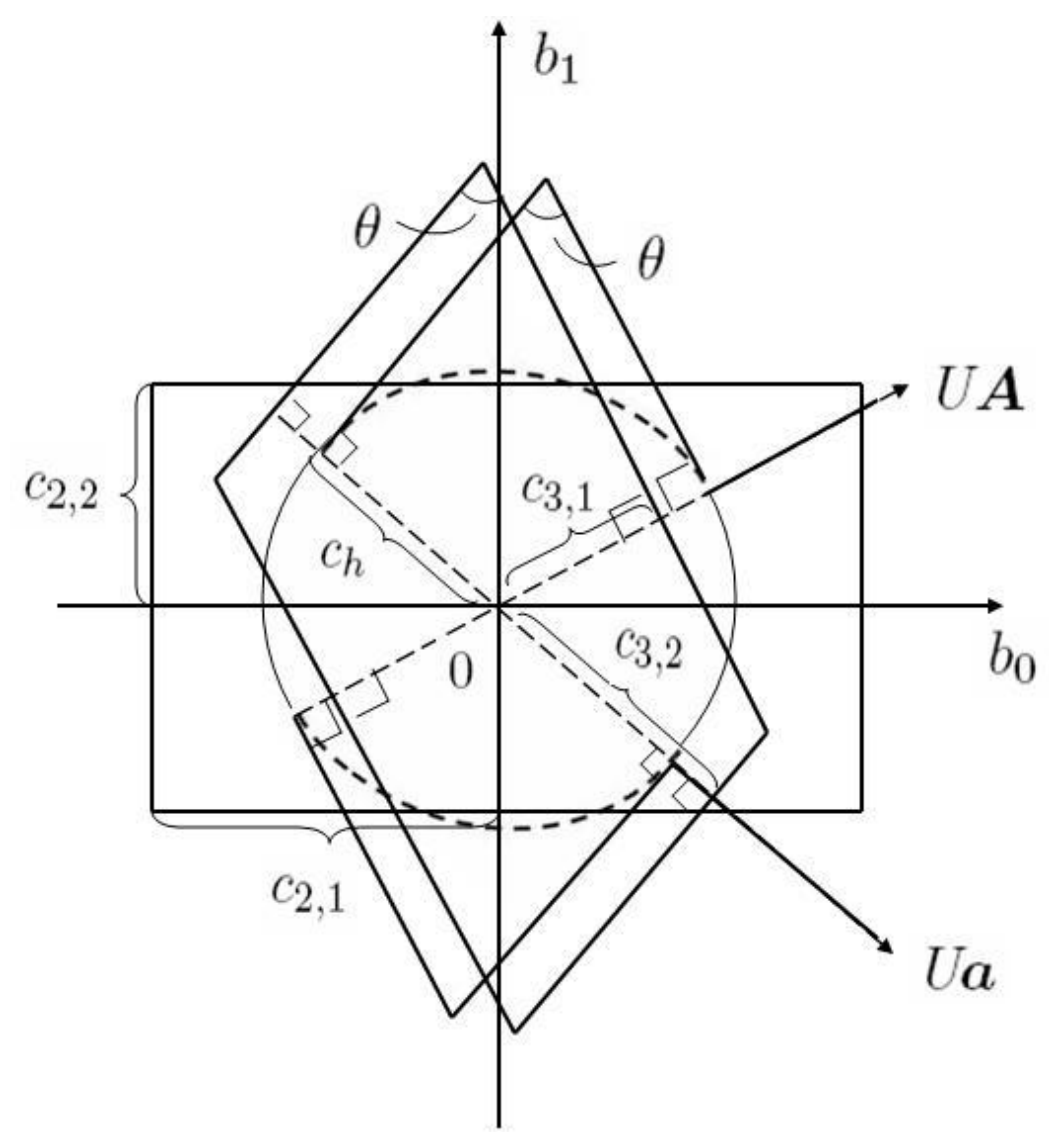


Figure 2: A picture used in the proof of Lemma 3.

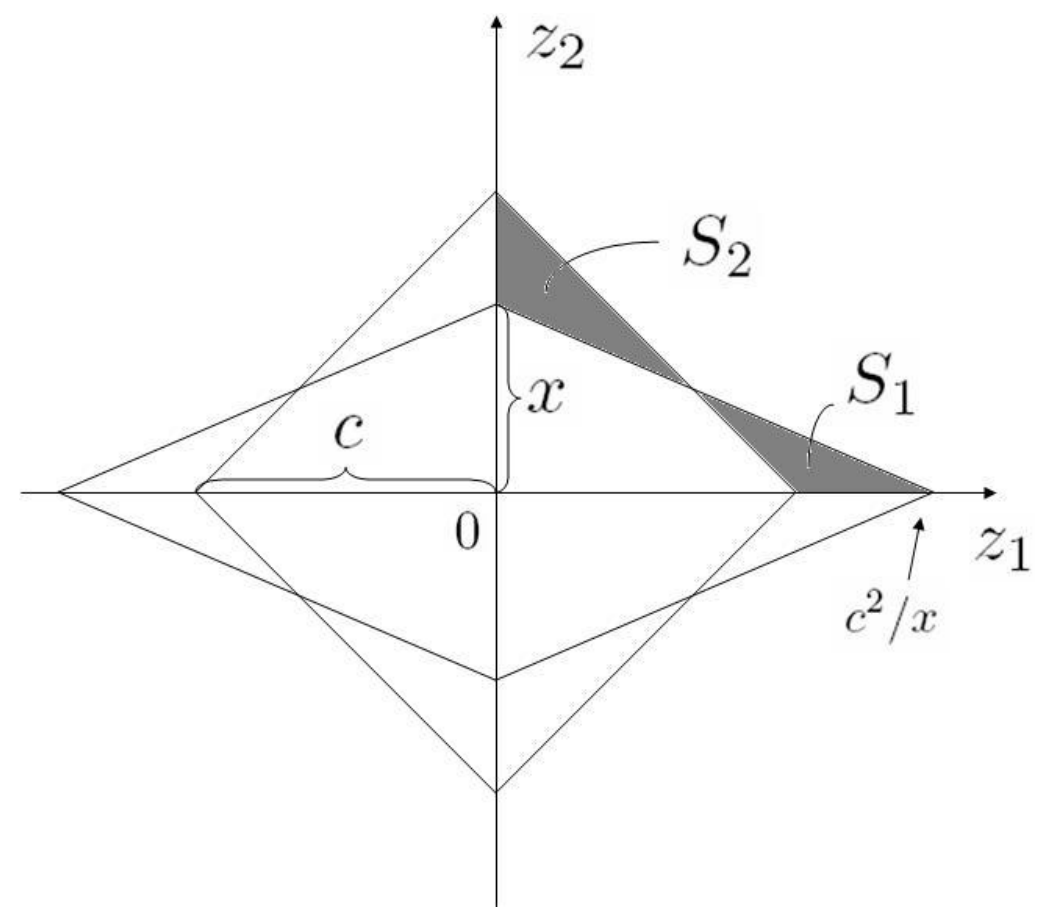


Figure 3: The spindle $R_{h}$ and rhombus $R_{3}$ used in the section 5 .

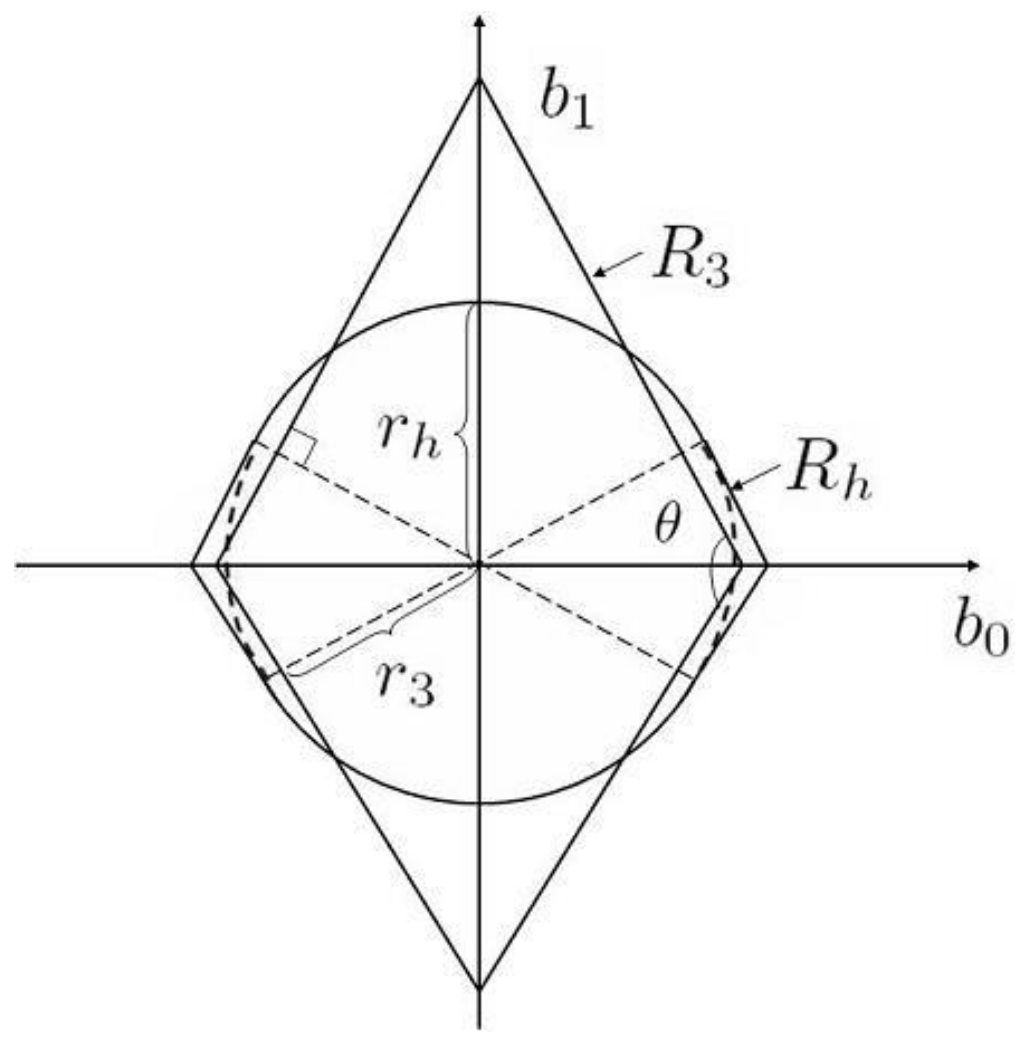


Figure 4. A plot of the function $e(\theta)$. The diamond on the curve identifies the point at which $e(\theta)=1$

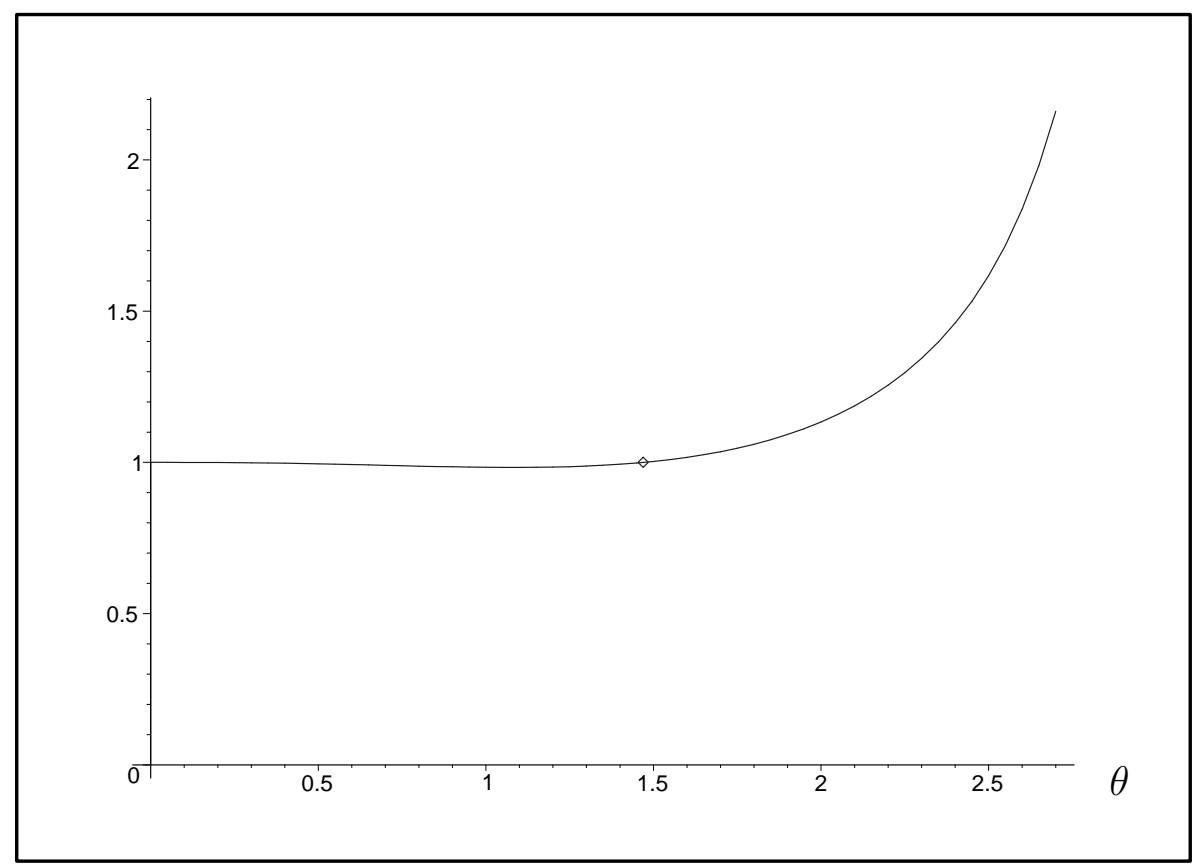

Figure 5. The data points, fitted line and several confidence bands. The hyperbolic band is given by the two solid curves, the two and three segment bands are identified in the picture.

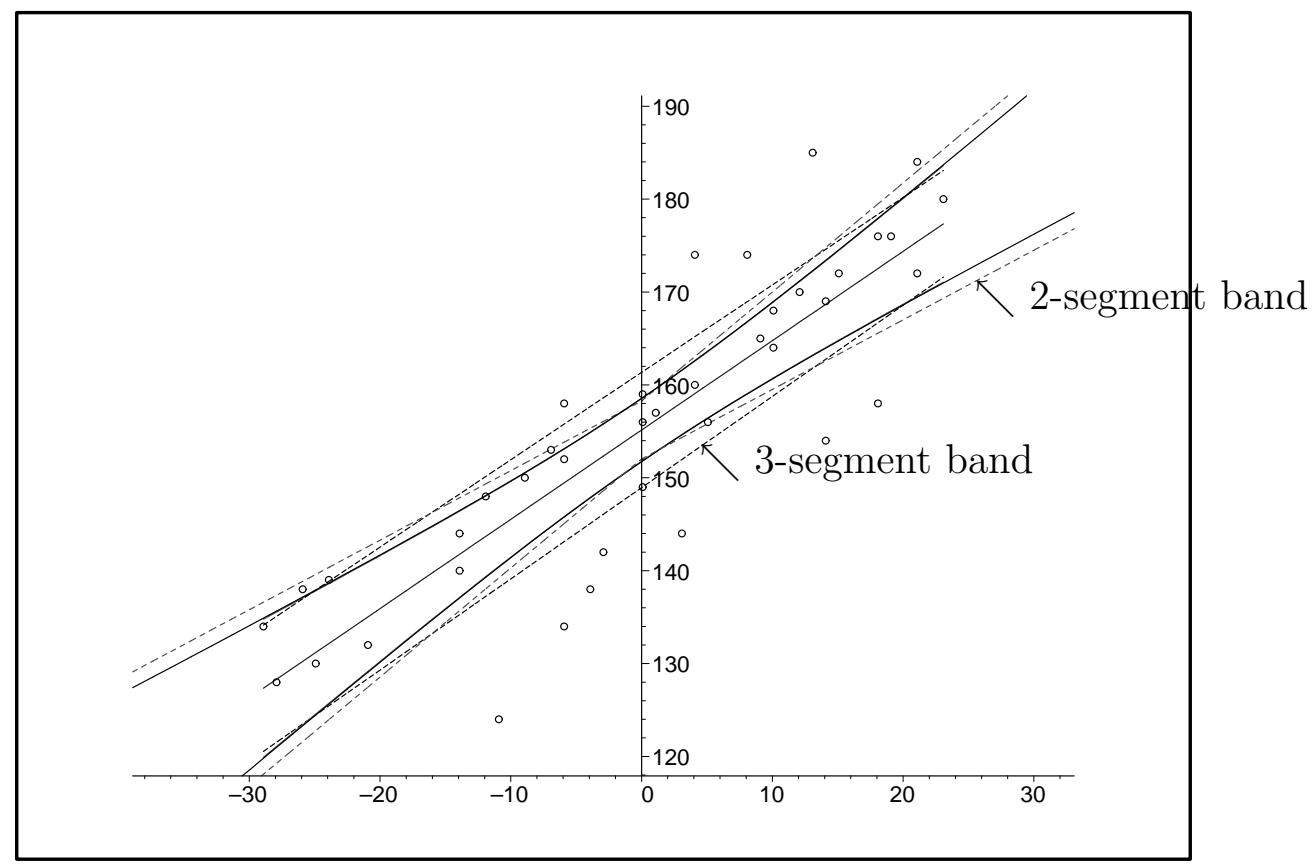


Figure 6. The $R$-regions of several confidence bands for the example in Section 6: the square - the best two-segment band; the circle - the Scheffé band; the rhombus - the best three-segment band; the spindle - the hyperbolic band over the interval $(-28.92,23.08)$.

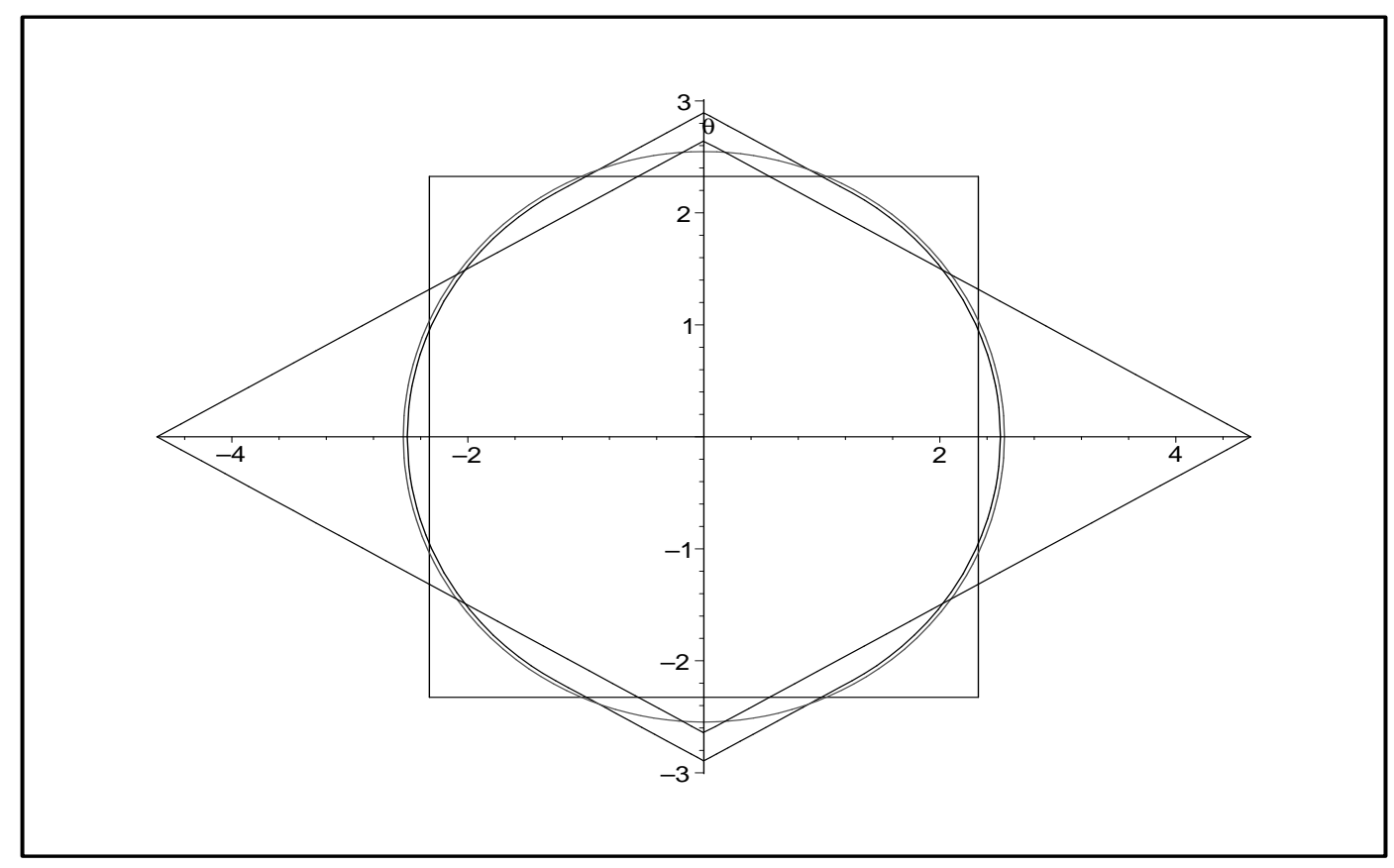

